\title{
Mean R Wave Amplitude
}

National Cancer Institute

\section{Source}

National Cancer Institute. Mean R Wave Amplitude. NCI Thesaurus. Code C62136.

The average (mean) amplitude (usually in $\mathrm{mm}$ ) of the $\mathrm{R}$ wave, obtained from a set of measurements of the R wave, in a particular lead or set of leads. 